\title{
Lung Manifestations of COVID-19 on Chest Radiographs_Indian Experience in a High-Volume Dedicated COVID center
}

\author{
Bhagyam Nagarajan $^{1} \cdot$ Gayatri Autkar $^{2} \cdot$ Aarav Monga $^{3} \cdot$ Nikhil Toshniwal $^{1}$
}

Accepted: 9 November 2020 / Published online: 6 January 2021

(C) Springer Nature Switzerland AG 2021

\begin{abstract}
Chest imaging, which includes X-ray imaging and CT scan, is the main modality for assessing lung involvement in patients affected with the COVID-19 virus. Although CT is more sensitive, due to ease and affordability issues, X-rays are the preferred first-line study. The aim of this article is to familiarize the treating physician with the imaging spectrum of the coronavirus lung infection on X-ray and to discuss the frequency of these findings. A total of 593 radiographs of admitted COVID-19 patients (RT-PCR proven) were retrospectively assessed in the study. Demographics of admitted patients and COVID manifestations on chest radiographs were assessed. Male to female ratio of patients in our study was 2.1:1. The largest number of patients was in the 50 to 60 -year age bracket (29\%). Forty percent of the X-rays in our study were negative. No X-ray showed findings exclusively in the upper zones, and $88 \%$ showed findings in the mid-lower zones. Ground glass opacification was the commonest finding (75\% of cases) in abnormal X-rays. The next most common findings were peripheral lung opacities and confluent consolidation. Confluent consolidation, which indicates more severe disease, was observed in $15 \%$ of the abnormal X-rays. The proportion of patients showing confluent consolidation was seen more in the older age groups ( $>50$ years old) with a peak in the 60-70-year age bracket. Small reticular opacities, pneumothorax, pneumomediastinum, and pleural effusions were uncommon findings in our study.
\end{abstract}

Keywords COVID-19 $\cdot$ X-ray $\cdot$ Consolidation $\cdot$ Peripheral lung opacity $\cdot$ Pneumothorax

\section{Introduction}

Although COVID-19 infection primarily affects the respiratory tract, it is now increasingly being recognized as more than an interstitial pneumonia. Associated cardiac, renal, and neurological findings have been described in some patients [1]. Some of the common clinical features of COVID-19 include cough, fever, fatigue, sore throat, and shortness of breath [2].

This article is part of the Topical Collection on Covid-19

Bhagyam Nagarajan

bhagyam.nagarajan@wockhardthospitals.com

1 Department of Radiology, Wockhardt Hospitals, 1877, Doctor Anandrao Nair Marg, Mumbai Central,

Mumbai, Maharashtra 400011, India

2 Department of Radiology, Mayo Hospital, Indira Gandhi Government Medical College, Central Ave, Mominpura, Nagpur, Maharashtra 440018, India

3 Dhirubhai Ambani International School, Trident Road, G Block BKC, Bandra Kurla Complex, Bandra East,

Mumbai, Maharashtra 400098, India
Important diagnostic tests for COVID-19 that have emerged are the nucleic acid amplification tests and imaging modalities of chest X-ray and CT scan. Due to infection control and affordability issues, chest $\mathrm{X}$-ray is considered the first-line imaging test in COVID-19 infection [2]. The presenting chest $\mathrm{X}$-ray findings aid the initial assessment, monitoring, and therapy of COVID-19 positive patients. Wockhardt Hospital in Mumbai, India, where the study has been conducted, was converted into an exclusive COVID center earlier this year, in the midst of the COVID-19 pandemic. Till date, almost 1500 COVID-19 positive patients have been treated at our hospital. Our aim was to retrospectively review the X-rays of proven COVID-19 patients and describe the common and less common radiographic features.

\section{Methods}

One thousand one hundred X-rays pertaining to 563 unique and RT-PCR proven COVID-19 positive patients admitted in Wockhardt Hospital, Mumbai, between May 10 and June 30, 2020 , were assessed by us. Institutional approval was obtained 
for the same. The initial X-rays of 563 unique patients were included in the study. In addition to the initial x-rays, $30 \mathrm{X}$ rays from 22 of these patients where there was significant radiological progression within 5-6 days of initial presentation were also included. Technically suboptimal follow-up Xrays were excluded. Also, follow-up X-rays of patients where the features were stable or did not show significant radiological progression were excluded from the study. Thus, a total of 593 X-rays were included in the study.

Methodology flow chart

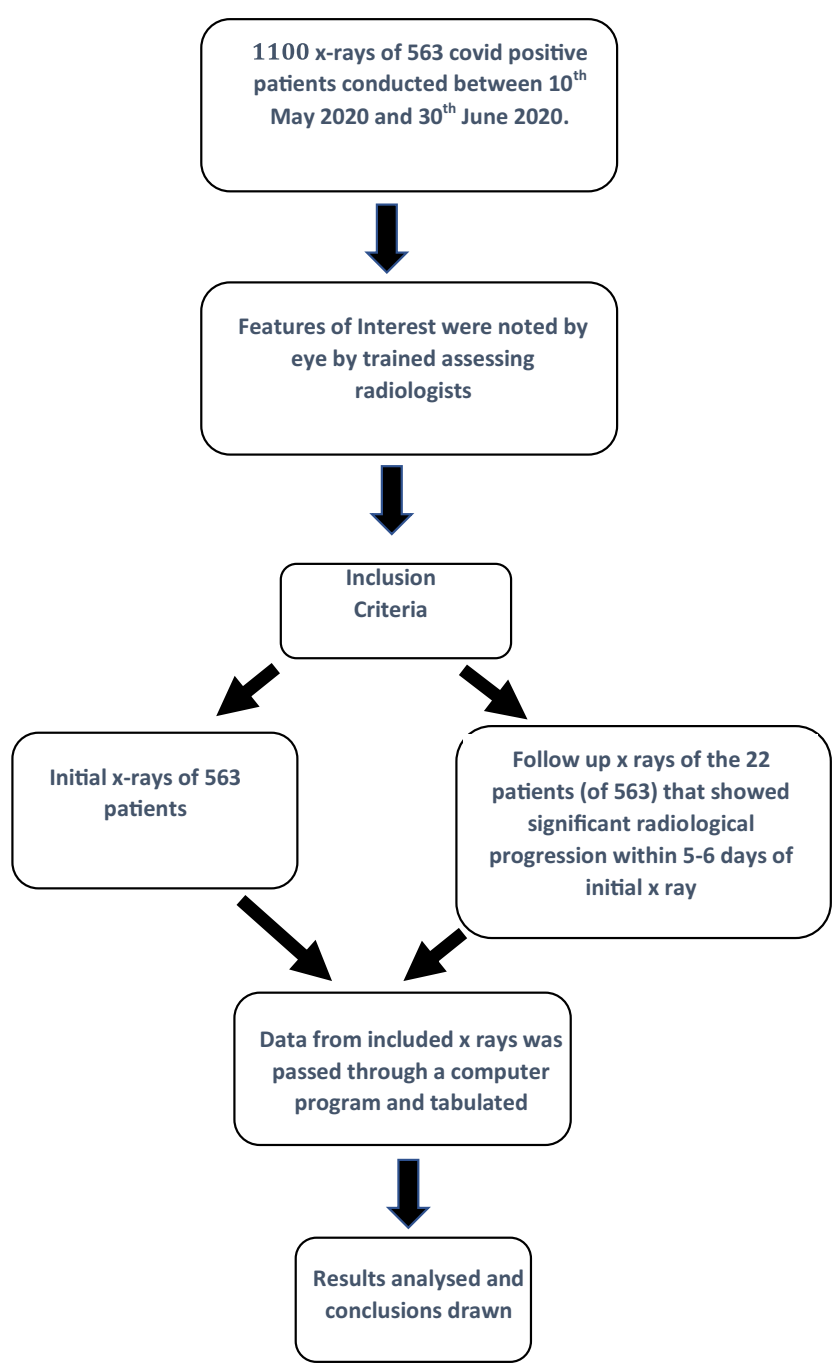

All the X-rays reviewed were portable X-rays in an anteroposterior (AP) view taken using a Siemens Multimobil 2.5 machine with exposures of $70-80 \mathrm{kVp}$ and $20 \mathrm{mAs}$. Patient position was either supine or sitting depending on patient morbidity. X-ray findings were reviewed by two radiologists (with combined 25 years of experience). Presence and features of lung involvement, laterality, and zonal distribution of findings were assessed. The lung zones were demarcated on the chest AP view as follows: the upper zone was extending from the lung apex to the superior border of the hilum, the lower zone was taken from the inferior hilar border to the lung base up to the costophrenic sulcus, and the mid zone was the portion in between the upper and lower zones.

X- ray features observed (Figs. 1 and 2) were categorized as follows:

1. Ground glass opacification (GGO)

2. Peripheral lung opacity (PLO)

3. Confluent consolidation

4. Reticular/irregular opacities

5. Pneumothorax

6. Pneumomediastinum

7. Pleural effusion

8. Adenopathy

$\mathrm{X}$-rays findings varied from none, one, to multiple features.

Some of the difficulties in reporting lung findings were related to patient position and obesity. The left lower zone on chest X-ray was sometimes obscured due to overlying cardiac shadow or due to a slight rotation of the patient. In these cases, the X-rays were read by both radiologists, arriving at a common consensus.

In many radiographs, GGO was present in the mid-lower zones and easy to diagnose due to the lucency of the uninvolved upper zones. In some, the ground glass haziness was more in one lung and hence was easy to spot. The challenge was reporting GGO with certainty when there is diffuse haziness in both lungs, especially in obese patients. In these cases, after adjusting technical factors such as contrast, lung lucency was compared with tracheal lucency and stomach bubble to differentiate apparent haziness from actual GGO.

\section{Results and Discussion (See Table 1) \\ Demographics}

Three hundred eighty-one of the 563 patients were male with a male to female ratio of 2.1:1.

The majority of patients were above 40 years of age. Only 2 patients were less than 20 years of age. The largest number of patients was in the 50-60-year age bracket.

\section{Radiological Findings}

Two hundred thirty-five of 593 X-rays were negative showing that a significant number of symptomatic COVID patients (40\%) may have normal X-rays (Table 1). 
Fig. 1 Chest X-ray AP portable in 4 different patients showing different lung findings in COVID-19 infection. Technical factors: $70 \mathrm{Kv}$ and $20 \mathrm{mAs}$. Image a shows normal lungs. Image $\mathbf{b}$ shows peripheral consolidation on the right and peripheral lung opacities on the left. Image c shows peripheral ground glass opacification in mid and lower zones. Image $\mathbf{d}$ shows typical "batwing" appearance of peripheral consolidation

Fig. 2 Chest X-ray AP portable in 2 patients showing radiological progression. Technical factors: $70 \mathrm{kV}$ and $20 \mathrm{mAs}$. Images a and b show generalized confluent consolidation of both lungs on a background of ground glass haziness with additional features of pneumothorax and pneumomediastinum. Images c and $\mathbf{d}$ show bilateral intercostal drains for pneumothorax and extensive subcutaneous emphysema. An additional feature of pneumomediastinum is also seen in image $\mathbf{d}$

\section{a}

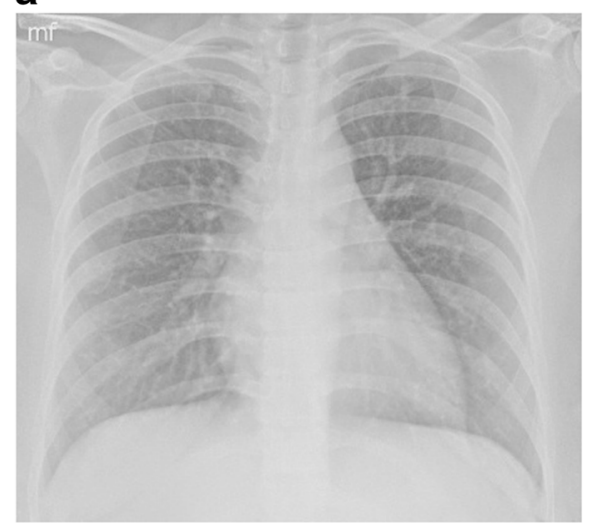

C

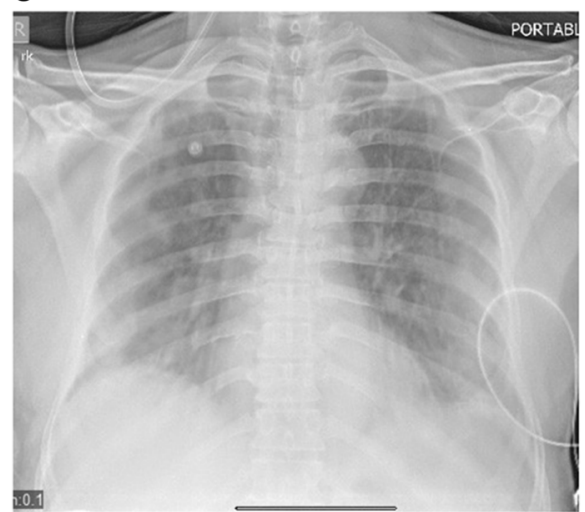

a

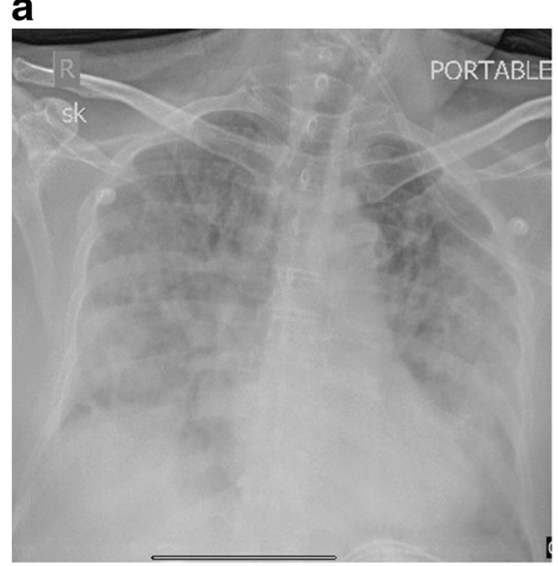

c

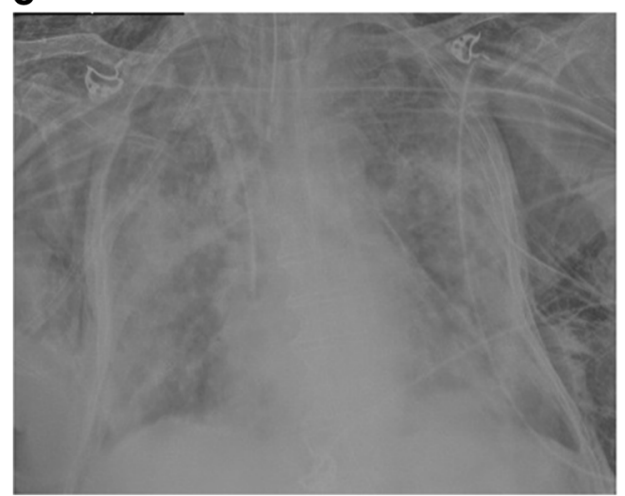

b

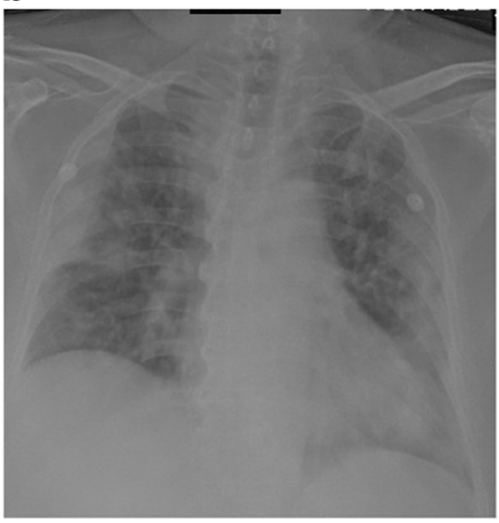

d

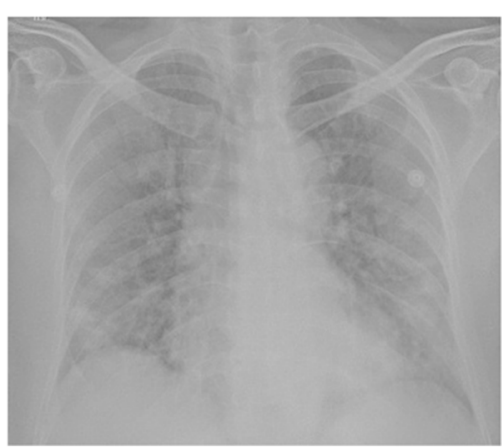

b

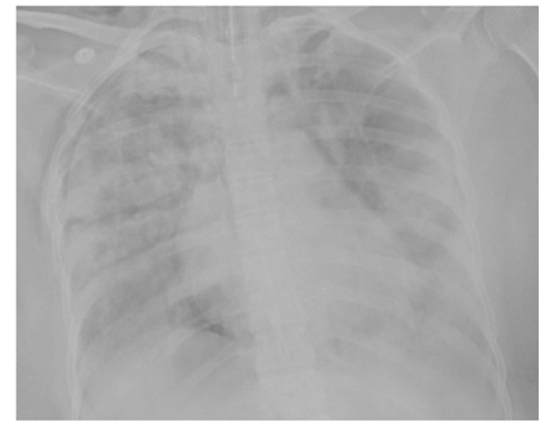

d

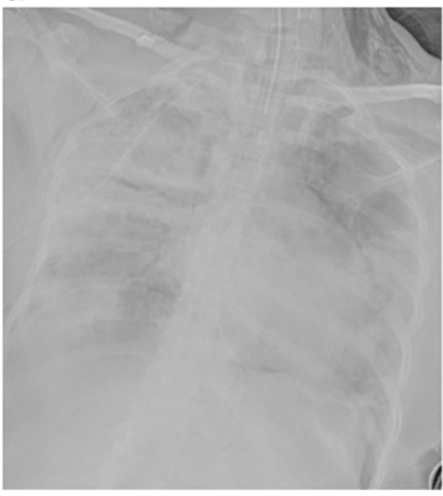


Table 1 Radiological findings of COVID-19 on chest radiographs

\begin{tabular}{|c|c|c|}
\hline Features & Number of Findings & $\%$ of X-rays with findings \\
\hline \multicolumn{3}{|l|}{ 1. Radiographical findings } \\
\hline Features present & 358 & 60.4 \\
\hline Features absent & 235 & 39.6 \\
\hline \multicolumn{3}{|c|}{ Sections $2,3,4,5$, and 6 pertain to the $358 \times$ rays with features present } \\
\hline \multicolumn{3}{|c|}{ 2. Laterality findings of $358 \mathrm{X}$-rays } \\
\hline Unilateral & 144 & 39.9 \\
\hline Bilateral & 217 & 60.1 \\
\hline \multicolumn{3}{|l|}{ 3. Zonal distribution } \\
\hline Upper region & 0 & 0 \\
\hline Mid-lower region & 314 & 87.7 \\
\hline Full lung & 44 & 12.3 \\
\hline \multicolumn{3}{|l|}{ 4. Radiographical findings } \\
\hline Ground glass opacification & 269 & 75.1 \\
\hline Confluent consolidation & 53 & 14.8 \\
\hline Peripheral lung opacities & 88 & 24.6 \\
\hline Reticular/irregular opacities & 13 & 3.63 \\
\hline Pneumothorax & 2 & 0.56 \\
\hline Pneumomediastinum & 1 & 0.28 \\
\hline Pleural effusions & 1 & 0.28 \\
\hline Adenopathy & 0 & 0 \\
\hline \multicolumn{3}{|c|}{ 5. Age distribution of patients with chest X-rays showing confluent consolidation } \\
\hline$<20$ years & 0 & 0 \\
\hline 20-30 years & 0 & 0 \\
\hline $30-40$ years & 2 & 3.77 \\
\hline $40-50$ years & 10 & 18.9 \\
\hline $50-60$ years & 17 & 32.1 \\
\hline $60-70$ years & 14 & 26.4 \\
\hline$>70$ years & 10 & 18.9 \\
\hline \multicolumn{3}{|c|}{ 6. Age distribution of patients with chest $\mathrm{X}$-rays showing full lung distribution } \\
\hline$<20$ years & 0 & 0 \\
\hline $20-30$ years & 0 & 0 \\
\hline $30-40$ years & 6 & 13.6 \\
\hline $40-50$ years & 8 & 18.1 \\
\hline $50-60$ years & 12 & 27.2 \\
\hline $60-70$ years & 13 & 29.5 \\
\hline$>70$ years & 5 & 11.4 \\
\hline
\end{tabular}

Bilateral findings were seen in $217(61 \%)$ of the $358 \mathrm{X}$-rays that were abnormal. The remaining 144 (39\%) X-rays showed unilateral findings.

None of the radiographs showed findings in the upper zones only. Three hundred fourteen of the $358 \mathrm{X}$-rays with findings showed mid-lower zone distribution. The remaining $12 \%$ showed findings in the full lung.

Sixty percent of the total X-rays were abnormal and showed unilateral or bilateral lung manifestations which included ground glass opacification, peripheral lung opacities, confluent consolidation, and reticular or small irregular opacities (Table 1). Additional features such as pneumothorax, pneumomediastinum, pleural effusion, and adenopathy were seen uncommonly. GGO (Fig. 1), either unilateral or bilateral, appeared most frequently in the abnormal X-rays ( $75 \%$ of abnormal x rays). In $81 \%$ of the $269 \mathrm{X}$-rays which showed GGO, GGO was an exclusive finding. PLO (Fig. 1) was next in frequency of appearance and seen in $88 \mathrm{X}$-rays (25\%). Confluent consolidation (Figs. 1 and 2) was seen in 15\% of the chest X-rays with findings. Pneumothorax and pneumomediastinum (Fig. 2) were present in only 3 out of the abnormal $358 \mathrm{X}$-rays (< $1 \%$ ), as were pleural effusions. Adenopathy was not present in any chest X-ray. 
Fig. 3 Histogram showing distribution of chest X-rays with confluent consolidation by age of patients, as a $\%$ of size of the age bracket

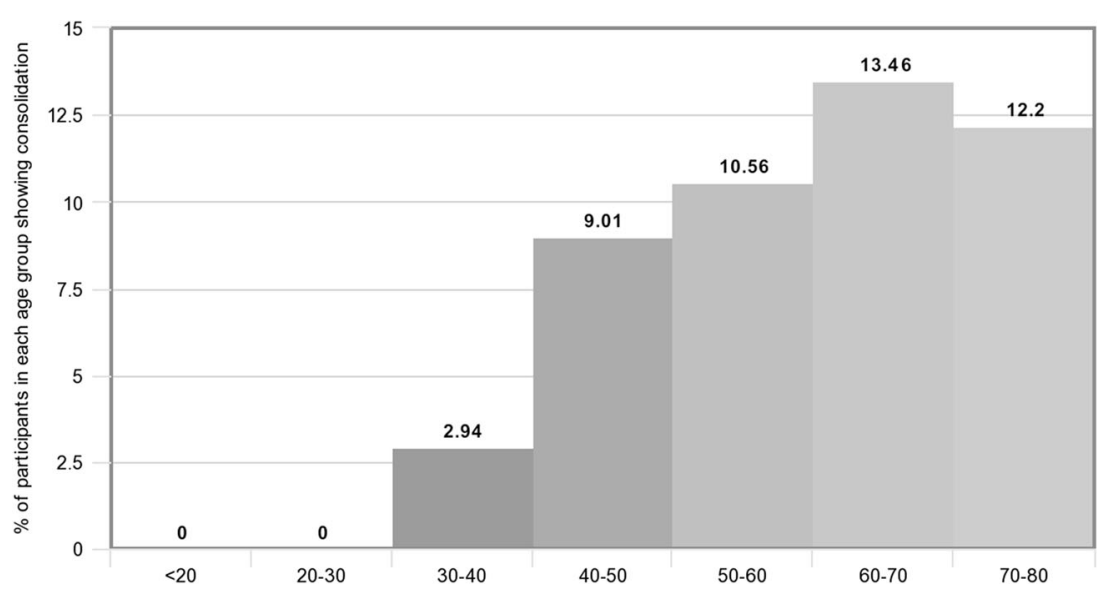

Confluent consolidation and full lung involvement have been used as markers for severe cases of COVID-19 in the lung. The age distribution of patients with confluent consolidation in the lung(s) is negatively skewed showing greater distribution in older age groups, with a visible increasing trend as age rises and a peak in the 60-70-year age bracket (Fig. 3, Table 1). To account for variations in age demographics of the study, the number of patients showing confluent consolidation has been expressed as a $\%$ of the total number of patients in that age group.

Forty-four patients showed full lung involvement. Of these, 30 patients were older than 50 years of age with the maximum number of patients (13) in the age bracket of 60-70 years. There were no patients with whole lung findings less than 30 years of age (Table 1).

\section{Discussion}

Our study of 593 X-rays of COVID patients is one of the larger studies that has been undertaken in the recent past. There were no X-rays with only upper zone involvement in our study which agrees with the previous article by Wong et al. [3]. Sixty percent of abnormal X-rays in our study showed bilateral findings as seen generally in viral pneumonias [4].

In our study, consolidation was further divided into patchy peripheral lung opacity (PLO), reticular opacity, and confluent consolidation similar to study published by Jacobi et al. [5]. Only $14 \%$ had confluent consolidation in our study which denotes severe disease. Reticular or irregular small opacities were seen only in $3.6 \%$ of abnormal cases. Also, in all cases, these were not an exclusive finding and were in conjunction with other findings such as GGO.

Our study shows a clear trend of increasing severity of lung involvement in COVID infection with age, with a peak at ages 60-70. This is in concordance with study by Hosseyni et al.
[4] which also showed older age and progressive consolidation suggesting poorer prognosis.

Subcutaneous emphysema, pneumothorax, or mediastinal emphysema, either spontaneous or after intubation in the setting of COVID-19 infection, has been described [6,7]. Our study included 2 cases with pneumothorax and 1 with associated pneumomediastinum after intervention. No X-rays showed adenopathy, and only 1 showed pleural effusion.

\section{Conclusion}

Our retrospective analysis of radiographic features in COVID19 patients was conducted on a high-volume data set of 593 $\mathrm{X}$-rays of COVID positive patients.

In our analysis, lung manifestations were primarily found in participants $>50$ years of age $(62 \%)$.

The majority of the patients were males $(68 \%)$.

Ground glass opacification was the most common finding ( $75 \%$ of X-rays with findings) and was seen early on in the disease.

Confluent consolidation was suggestive of progression and was reflected in $15 \%$ of X-rays.

When confluent consolidation was used as a metric for severity, higher age groups were revealed to be more susceptible to serious progression. The maximum proportion of patients showing confluent consolidation was in the age group $60-70$ years (13.46\% of total age group).

When the zonality of COVID-related findings (full lung(s)) was used as a metric for severity, the age group with the highest proportion of $\mathrm{X}$-rays with severe findings was again shown to be $60-70$ years.

Rarer findings included reticular/irregular opacities, pneumothorax, and pneumomediastinum.

No X-rays showed adenopathy; only 1 showed pleural effusion. Thus, these findings are rare in COVID unless there is a co-existing bacterial disease. 
Acknowledgments We would like to thank Wockhardt Hospital's X-ray technicians, doctors, and staff who have dedicated their time and efforts to treat the COVID-19 patients.

Authors' Contribution Conceptualization, writing original draft, data collection, and interpretation and supervision were carried out by corresponding author and second author. Data analysis, evaluation and interpretation of data, discussion, editing, and image formatting were carried out by the third author. Resource materials, initial draft, and editing were done by the fourth author.

Data Availability All data supporting this manuscript are available upon request to the corresponding author.

\section{Compliance with Ethical Standards}

Conflict of Interest The authors declare that they have no conflict of interest.

Ethics Approval The study complied with the guidelines laid out by the Hospital Ethics Committee.

Consent to Participate Not applicable as this is a retrospective study of images.

Consent for Publication Not applicable.

Code Availability Not applicable.

\section{References}

1. Spuntarelli V, Luciani M, Bentivegna E, Marini V, Falangone F, Conforti G, et al. Covid-19: is it just a lung disease? SN Compr Clin Med. 2020;2:1401-6.

2. Harahwa TA, Lai Yau TH, Lim-Cooke MS, al-Haddi S, Zeinah M, Harky A. The optimal diagnostic methods for COVID-19. Diagnosis (Berl). 2020;0. https://doi.org/10.1515/dx-2020-0058.

3. Wong HYF, et al. Frequency and distribution of chest radiographic findings in COVID-19 positive patients. Radiology. 2020. https:// doi.org/10.1148/radiol.2020201160.

4. Hosseiny, et al. Coronavirus disease 2019(COVID-19): lessons from severe acute respiratory syndrome and Middle East respiratory syndrome. AJR. 2020;214:1078-82.

5. Jacobi A, Chung M, Bernheim A, Eber C. Portable chest X-ray in coronavirus disease-19 (COVID-19): a pictorial review. Clin Imaging. 2020;64:35-42.

6. Romano N, Fischetti A and Melani EF. Pneumomediastinum related to Covid-19 Pneumonia. Am J Med Sci. 2020. https://doi.org/10. 1016/J.AMJMS.2020.0603.

7. Sun R, Liu H, Wang X. Mediastinal emphysema, Giant Bulla, and pneumothorax developed during the course of COVID-19 pneumonia. Korean J Radiol. 2020;20.

Publisher's Note Springer Nature remains neutral with regard to jurisdictional claims in published maps and institutional affiliations. 